\title{
A multi-objective approach for the clustering problem in wireless ad hoc networks
}

\author{
Khalil Amine* \\ MOAD6 Research Team, Mohammadia School of Engineering, Mohammed V University of Rabat, Morocco
}

\begin{abstract}
Ad hoc networks are infrastructure-less computer networks consisting of heterogeneous mobile devices connected by wireless links. The devices are essentially faced to several limitations in terms of embedded energy, processing power, bandwidth, and internal memory. Clustering has been introduced in ad hoc networking to provide hierarchy and stability and to support information circulation reduction, energy management, and scalability. It consists of a process of self-aggregation of nodes into a set of sub-networks called clusters where each cluster would be managed by a master node called cluster centre responsible for gathering, maintaining and updating the cluster topology information as well as ensuring the data routing over the network. The set of cluster centres institutes a temporary network core that enhances the topology maintenance. From a graph perspective, this set represents a dominating set, that is a set of nodes such that each node of the network is either an element or adjacent to at least one element of this set. This paper develops a constrained bi-objective optimisation model for finding a dominating set that provides a trade-off between maximising the energy amount and minimising the cardinality. A bi-objective simulated annealing meta-heuristic for constructing near-optimal solutions is then presented.
\end{abstract}

\section{Introduction}

An ad hoc network is a wireless infrastructure-less network containing heterogeneous mobile devices with several limitations in terms of embedded energy, processing power, bandwidth, and internal memory. Ad hoc networks are self-organising networks: nodes are able to establish reactively a temporary network topology tolerant of any increase or decrease in the number of devices, and are able to find multi-hop routes to relay any message, when needed. Typical applications of ad hoc networks include military intervention, emergency operations, remote areas coverage, and vehicular communication networks.

Ad hoc networks are faced to two main challenges, namely the energy management and the dynamic topology. Clustering has been introduced in ad hoc networking to deal with such situation. It consists of a process of self-aggregation of nodes into a set of sub-networks called clusters where each cluster would be managed by a master node called cluster centre responsible for gathering, maintaining and updating the cluster topology information as well as ensuring the data routing. Clustering allows reducing the total network energy consumption by decreasing the amount of 'circulatory' information, and limiting nodes responsibility. It brings temporary network core that enhances the topology stability and increases the network lifetime.

From a graph theoretical perspective, cluster centres represent a dominating set, that is a set of nodes such that each node of the network is either an element or adjacent to at least one element of this set. Selection of cluster centres is the main stage of a clustering process that requires a specific selection criterion. This paper develops a constrained bi-objective optimisation model for finding a dominating set that provides a trade-off between maximizing the energy amount and minimising the cardinality. A bi-objective simulated annealing

*e-mail: khalilamine@research.emi.ac.ma meta-heuristic for constructing near-optimal solutions is then presented.

\section{Background and problem statement}

Clustering in ad hoc networks consists of a self-aggregation of nodes into a number of sub-networks called clusters. Each cluster is managed by a master node called cluster centre, or more commonly cluster head. Clustering institutes an abstract structure, as illustrated in figure 1, that allows to differentiate between three types of nodes:

- cluster centre, responsible for gathering, maintaining and updating the cluster topology information as well as ensuring data routing;

- ordinary member, a node related to a cluster centre;

- gateway, a router node enabling the access to its related cluster.

This temporary structure enhances the network stability and increases the network lifetime. Furthermore, it allows reducing the amount of circulatory information, thereby reducing the total network energy consumption. From a conceptual perspective, while the communication is managed proactively inside clusters, each cluster can be considered as an independent entity allowing the communication over the network to be managed reactively. That is, information related to ordinary members is locally shared within the associated cluster and would be updated periodically, yet only information related to cluster centres is shared globally when needed.

Selection of cluster centres represents the main stage in a network clustering process that requires a specific selecting criterion. Several clustering criteria have been introduced in the literature, yet few studies $[5,13]$ have considered a selecting criterion that is related directly to the residual energy in the nodes. This work aims at dealing with energy limitation in ad hoc networks by considering the residual energy as main key of clustering. Accordingly, 


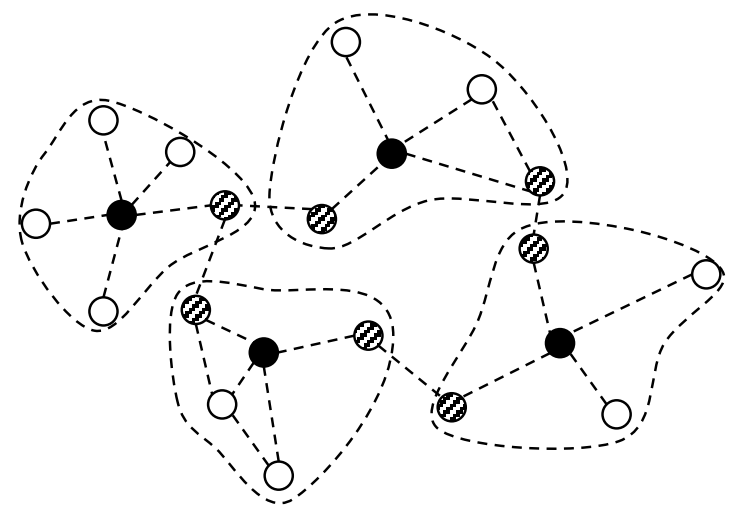

Cluster centre

Cluster member

Cluster gateway

-..- Wireless link

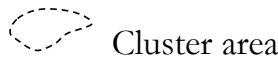

Figure 1: A hypothetical clustered topology

only nodes with high performance would be trusted to be assigned the cluster centre status. Besides, the number of selected cluster centres should be reduced as much as possible in order to preserve the total network energy amount.

From a graph theoretical perspective, clustering would consist of finding a dominating set, that is a set of nodes such that each node of the network is either an element or adjacent to at least one element of this set. Yet, as illustrated in figure 2, a dominating set for a graph is not unique and a network would contain many dominating sets with different cardinality and, then, with different performance. Therefore, considering an ad hoc network as an undirected graph, the considered problem is reduced to find an efficient dominating set that provides a compromise between the energy amount and the cardinality.

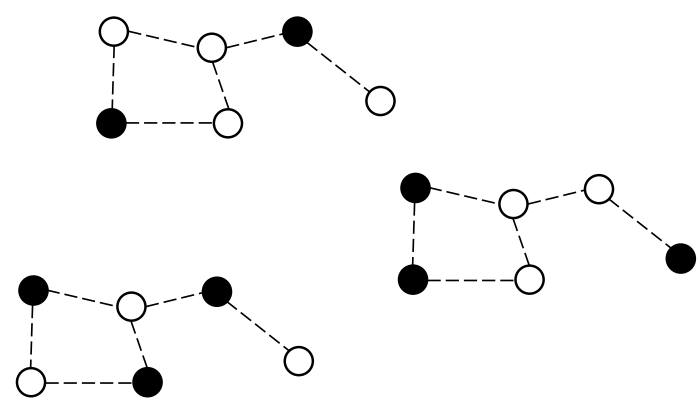

Figure 2: Three dominating sets for the same network

\section{Mathematical model}

Let $G=(V, E)$ be an undirected graph where $V$ is the set of vertices representing the network nodes and $E$ the set of edges (pairs of nodes) representing the available direct communication links, and let us consider the following notations

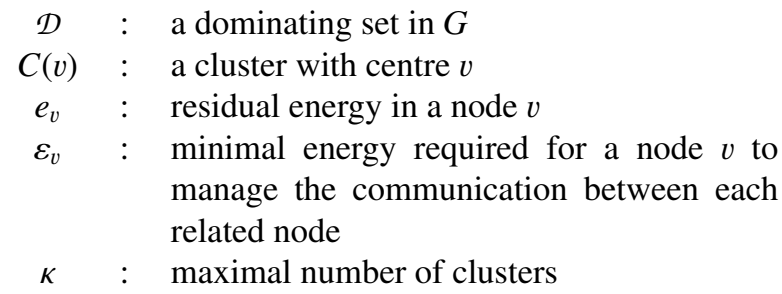

Let us consider the variables $x$ and $y$ such that

$$
x_{u v}= \begin{cases}1 & \text { if } u \in C(v) \\ 0 & \text { otherwise }\end{cases}
$$

and

$$
y_{v}= \begin{cases}1 & \text { if } v \text { is a cluster centre } \\ 0 & \text { otherwise }\end{cases}
$$

The proposed mathematical formulation consists of the following model

$$
\left\{\begin{array}{lll}
\max _{v} \sum_{v \in V} e_{v} y_{v} & & \\
\min _{v} \sum_{v \in V} y_{v} & & \\
\text { subject to } & & \\
\sum_{v \in V} x_{u v} & =1 & \forall u \in V \\
e_{v} y_{v} & \geq \varepsilon_{v} \sum_{u \in V} x_{u v} ; & \forall v \in V \\
\sum_{v \in V} y_{v} \leq \kappa & \leq\{0,1\} \quad ; \quad \forall u, v \in V
\end{array}\right.
$$

Model constraints explanation is given as follows:

- constraint (1) stipulates that each node is assigned to exactly one cluster centre;

- constraint (2) requires that each cluster centre should have enough energy to manage communications with all its cluster members;

- constraint (3) imposes a largest number $\kappa$ of constructed clusters.

Furthermore, we consider the following assumptions:

- the network is static or with low mobility;

- nodes have the ability to know their current connectivity degree;

- devices are equipped by an Advanced Configuration and Power Interface (ACPI) module (or similar) allowing managing and monitoring the embedded energy. In other words, nodes are able to access the information about their own residual energy;

- nodes are identified by means of a unique number (label): the Medium Access Control (MAC) address which is given by manufacturers to every wireless interface. 


\section{Proposed meta-heuristic}

Simulated annealing is a local search method introduced by Kirkpatrick et al. [6], initially for global combinatorial mono-objective optimisation problems. It consists at each iteration of generating a candidate solution that will be whether accepted as a result of a comparison with a current solution in hand. A certain transition rule is set according to the objective function (called energy function) that aims at moving regularly to an improving solution. Yet, some uphill solutions would be accepted occasionally in order to guarantee a diversity of choice within the domain exploration and to avoid getting caught at local optima. The number of iterations is controlled by a certain cooling schedule consisting of a temperature parameter and a decreasing function. Simulated annealing has showed significant success for many applications - in spite of the proven asymptotic convergence to the optimal solution, it allows gradual convergence to a near-optimal solution in practice, and it requires low short-term memory. More detailed review can be found in $[2,4,8,10]$.

Multi-objective simulated annealing approach has been introduced in the work of Serafini [7] and have merged in many subsequent works $[1,3,9,11,12$, etc.] providing a construction of the near-optimal solutions. In general, multi-objective simulated annealing methods

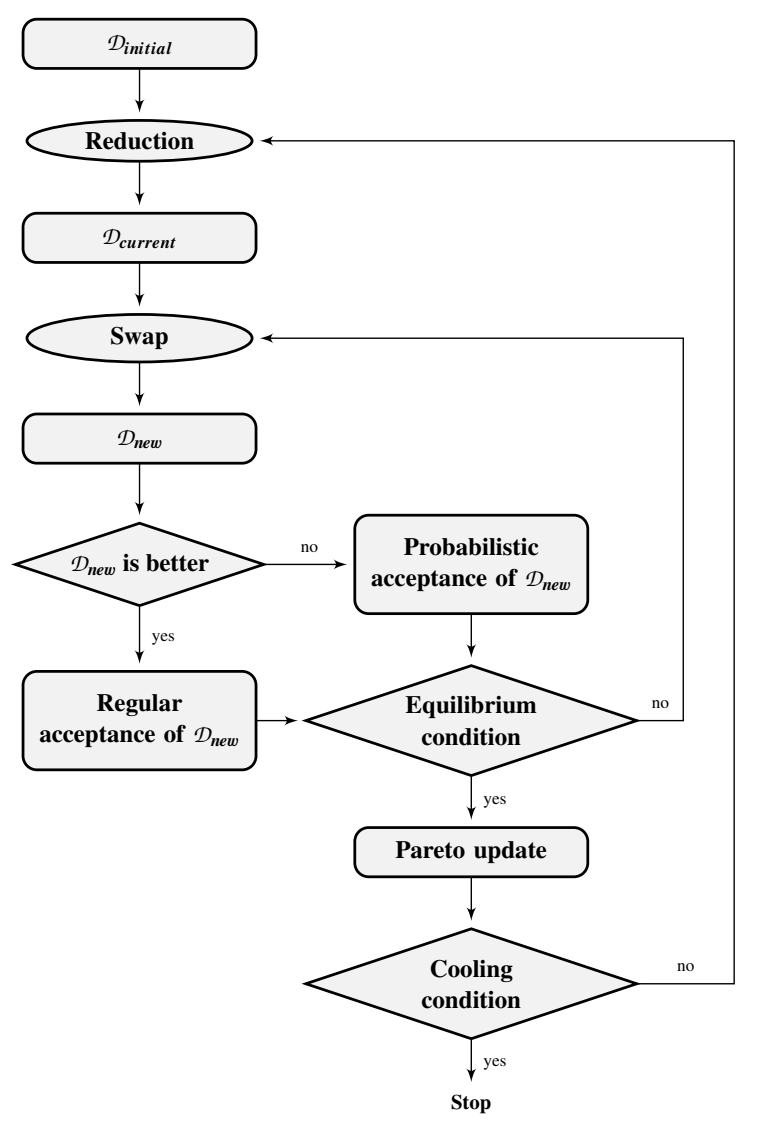

Figure 3: Proposed simulated annealing algorithm flowchart use an archive memory that catches the non-dominated solutions while exploring the feasible domain, and they often consider a variation of a certain composite energy function for solutions evaluation. Like all meta-heuristics, simulated annealing is problem-dependent and the choice of appropriate parameters is rather a challenging task, particularly in multi-objective case.

We propose a bi-objective simulated annealing approach to find dominating sets that each corresponds to a certain compromise between the total energy amount and the number of elements. As illustrated in figure 3, the meta-heuristic consists of two nested loops: the outer loop performs a cardinality reduction by removing a randomly chosen node from the input solution, and the inner loop consists of a swap procedure that generates neighbouring solutions by replacing randomly a node from the current solution by another one from the network.

The meta-heuristic starts from a solution of cardinality $\kappa$, and performs a sequence of reductions and swaps to result hopefully in an efficient solution for each cardinality level (cf. figure 4). The number of iterations is controlled by means of an equilibrium and a cooling condition. Some uphill solutions, possibly infeasible, would be accepted occasionally according to a probabilistic parameter that involves both the two objective functions. A 'Pareto archive' is set to store non-dominated solutions. Each efficient solution found is considered as a potential non-dominated solution for the current cardinality level and the Pareto archive is updated in consequence.

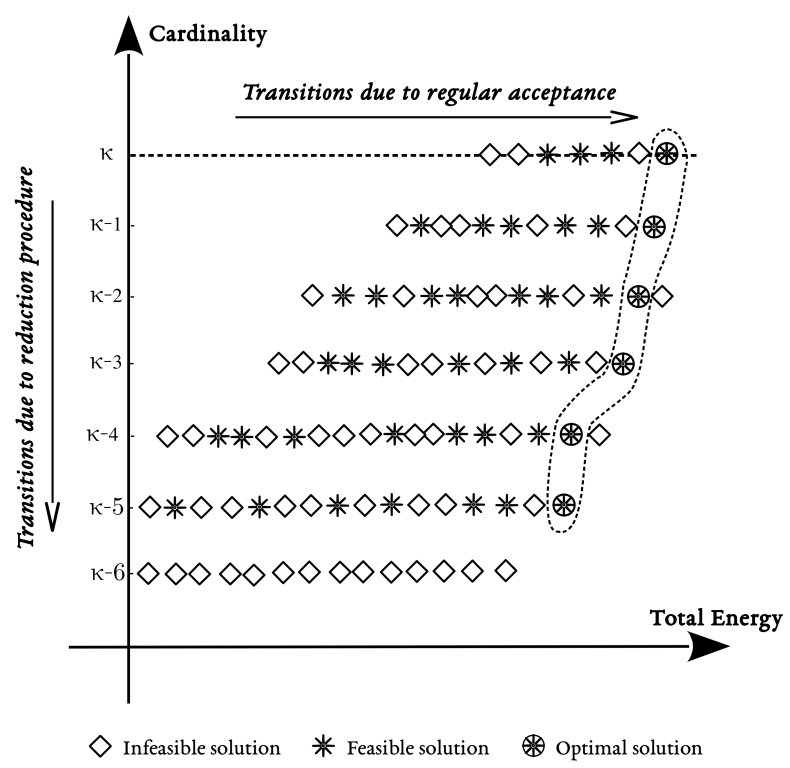

Figure 4: Directions of transitions in the objective space 


\section{Conclusion}

Clustering in ad hoc networks provides hierarchy and stability and supports information circulation reduction, energy management, and scalability. The main clustering stage is the selection of cluster centres that is equivalent from a graph theoretical perspective to finding a dominating set satisfying the considered clustering purpose. A constrained bi-objective optimisation model for finding a dominating set that provides a trade-off between maximizing the energy amount and minimising the cardinality has been introduced. Due to the significant success of simulated annealing and particularly for the requirement of low short-term memory, a bi-objective simulated annealing meta-heuristic for constructing near-optimal solutions has been proposed.

\section{References}

[1] M. H. Alrefaei and A. H. Diabat, "A simulated annealing technique for multi-objective simulation optimization," Applied Mathematics and Computation, vol. 215, no. 8, pp. 3029-3035, 2009.

[2] Kh. Amine, "Insights into simulated annealing," in Handbook of Research on Modeling, Analysis, and Application of Nature-Inspired Metaheuristic Algorithms, S. Dash, B. K. Tripathy, and A. Rahman, Eds. Hershey, PA, USA: IGI Global, 2018, pp. 121-139.

[3] S. Bandyopadhyay, S. Saha, U. Maulik, and K. Deb, "A Simulated Annealing-Based Multiobjective Optimization Algorithm: AMOSA," IEEE Transactions on Evolutionary Computation, vol. 12, no. 3, pp. 269-283, 2008.

[4] K. A. Dowsland and J. M. Thompson, "Simulated annealing," in Handbook of Natural Computing, G. Rozenberg, Th. Bäck, and J. N. Kok, Eds. Berlin Heidelberg: Springer-Verlag, 2012, pp. 1623-1655.

[5] D. Gavalas, G. Pantziou, Ch. Konstantopoulos, and B. Mamalis, "Clustering of mobile ad hoc networks: An adaptive broadcast period approach," in Proceedings of the $19^{\text {th }}$ IEEE International Conference on Communications, ser. ICC'06, vol. 9, Istanbul, Turkey, June 11-15 2006, pp. 4034-4039.

[6] S. Kirkpatrick, C. D. Gelatt, and M. P. Vecchi, "Optimization by simulated annealing," Science, vol. 220, no. 4598, pp. 671-680, 1983.

[7] P. Serafini, "Simulated annealing for multi objective optimization problems," in Multiple Criteria Decision Making: Proceedings of the Tenth International Conference: Expand and Enrich the Domains of Thinking and Application, G. H. Tzeng, H. F. Wang, U. P. Wen, and P. L. Yu, Eds. New York, NY: Springer-Verlag, 1994, pp. 283-292.

[8] N. Siddique and H. Adeli, "Simulated annealing, its variants and engineering applications," International Journal on Artificial Intelligence Tools, vol. 25, no. 6, 2016, 1630001, 25p.

[9] B. Suman, "Simulated annealing-based multiobjective algorithms and their application for system reliability," Engineering Optimization, vol. 35, no. 4, pp. 391-416, 2003.

[10] B. Suman and P. Kumar, "A survey of simulated annealing as a tool for single and multiobjective optimization," Journal of the Operational Research Society, vol. 57, no. 10, pp. 1143-1160, 2006.

[11] O. Tekinalp and G. Karsli, "A new multiobjective simulated annealing algorithm," Journal of Global Optimization, vol. 39, no. 1, pp. 49-77, 2007.

[12] E. Ulungu, J. Teghem, P. Fortemps, and D. Tuyttens, "MOSA method: A tool for solving multiobjective combinatorial optimization problems," Journal of Multi-Criteria Decision Analysis, vol. 8, no. 4, pp. 221-236, 1999.

[13] J. Wu, F. Dai, M. Gao, and I. Stojmenovic, "On calculating power-aware connected dominating sets for efficient routing in ad hoc wireless networks," Journal of Communications and Networks, vol. 4, no. 1, pp. 59-70, 2002. 